\title{
Benign Paroxysmal Positional Vertigo in Pregnancy
}

\author{
Kübra Çoban ${ }^{1}$, Nilüfer Yiğit ${ }^{2}$, Erdinç Aydın ${ }^{3}$ \\ ${ }^{1}$ Department of Otorhinolaryngology, Başkent University Alanya Research and Application Center, Antalya, Turkey \\ Case Report $>\begin{aligned} & { }^{2} \text { Department of Gynecology and Obstetrics, Başkent University Alanya Research and Application Center, Antalya, Turkey } \\ & { }^{3} \text { Department of Otorhinolaryngology, Başkent University Ankara Hospital, Ankara, Turkey }\end{aligned}$
}

\begin{abstract}
Benign paroxysmal positional vertigo (BPPV) is a clinical entity characterized by acute, brief paroxysmal attacks of rotational vertigo induced by head position changes. It is the most common peripheral vestibular pathology and is seen more frequently in women. However, to our knowledge, there is very limited data on the association between BPPV and pregnancy in both English
\end{abstract}

\section{Introduction}

Benign paroxysmal positional vertigo (BPPV) is a clinical entity characterized by acute, brief, paroxysmal attacks of rotational vertigo induced by head position changes. It is the most common peripheral vestibular pathology and is seen more frequently in women than men with a ratio of 2:1. In BPPV, degenerative debris dislocated from the utricle into the semicircular canals increase the density of the cupula (1). This may occur either when the deposits abnormally attach to the cupula (cupulolithiasis) or when the dense particles freely move in the endolymphatic fluid in the semicircular canals (canalolithiasis) (2).

A diagnosis of posterior semicircular canal BPPV is confirmed with a transient, upbeating, torsional nystagmus with the eyes beating toward the underlying ear when the patient is rapidly positioned into the lateral head-hanging position (Dix-Hallpike maneuver) (2). The most commonly used canalith-repositioning maneuver is the Epley maneuver (2-4). and Turkish literature. We present four pregnant women diagnosed with BPPV for the first time during gestation and revise the etiological factors of BPPV and the role of pregnancy-related changes in BPPV.

Keywords: Benign paroxysmal positional vertigo, pregnancy, gestation, vertigo
Address for Correspondence: Kübra Çoban E-mail: kubracob81@gmail.com Received Date: 16.11 .2016 Accepted Date: 31.01 .2017

(c) Copyright 2017 by Official Journal of the Turkish Society of Otorhinolaryngology and Head and Neck Surgery Available online at www.turkarchotorhinolaryngol.org DOl: 10.5152/ta0.2017.2079
The etiology may be idiopathic (50\%-70\% of all cases) or secondary to infections, migraine, $\mathrm{Me}-$ niere's disease, otologic/nonotologic surgery, prolonged bed rest, trauma, vascular, and metabolic pathologies, following magnetic resonance imaging (1-3, 5, 6). Giacomini et al. (1) discussed 10 cases of BPPV secondary to oral contraceptive use. They hypothesized that hormonal disorders in women may trigger some BPPV forms, and the increased prevalence in women compared with men could be associated with these hormonal alterations.

We present four pregnant women diagnosed with BPPV during their gestational periods. To our knowledge, very limited data are available in the English literature considering this co-occurrence. Three cases of pregnant women with BPPV were mentioned in a Chinese Journal; thus, this article could not be reviewed. We hope to inspire further clinical studies regarding this co-occurrence with our multiple case reports. Using this technique, we aimed to review the literature on BPPV etiology and discuss the possible pathophysiological factors contributing to BPPV in pregnancy. 


\section{Case Presentations}

\section{Case-1}

A 25-year-old woman pregnant for 29 weeks and 3 days attended the emergency clinic at our center with severe rotational vertigo and nausea. She experienced a sudden onset of vertigo with no other associated complaints of hearing loss, tinnitus, ear fullness, or pressure sense. Neurological status was stable. Pregnancy status was within normal limits. She had not previously been diagnosed with any peripheral or central audiovestibular pathologies. Physical examination findings were normal, except for a horizontal rotatory nystagmus in Dix-Hallpike right head-hanging position, which had a latency period of approximately 4-5 s and lasted less than a minute. Right posterior semicircular canal BPPV was diagnosed, and Epley maneuver was the treatment of choice as the canalith-repositioning maneuver. Symptoms were thus relieved, and there was no recurrence 10 months later.

\section{Case-2}

A 37-year-old woman pregnant for 33 weeks and 5 days visited our clinic with severe rotational vertigo, mild nausea, and vomiting. Vertigo was triggered with sudden head movements during sleep. She had no associated symptoms such as hearing loss, tinnitus, ear fullness, or pressure sense. She had not experienced any pregnancy-related problems. No co-morbid diseases were associated. She mentioned that her symptoms had started during the 16 th gestational week, which she underestimated, and the time of diagnosis was during her third vertigo attack. She had neither experienced any similar audiovestibular complaints nor been diagnosed with any peripheral vestibular pathologies.

Otorhinolaryngologic examination was within normal limits. Dix-Hallpike test was performed, and left horizontal rotatory nystagmus with a 2- to 3-s latency lasting for nearly $20 \mathrm{~s}$ was observed. Epley maneuver was performed. During the follow-up of 26 months, she experienced no further attacks.

\section{Case-3}

A 24-year-old woman pregnant for 19 weeks and 4 days was referred to our clinic with vertigo and nausea. Her symptoms had started nearly a month ago. Symptoms aggravated with sudden head movements, especially when lying down. She had no hearing loss, ear fullness, or tinnitus. Her pregnancy course was within normal limits. She had no chronic diseases, trauma, allergy, infection history, but low 25-hydroxyvitamin D levels.

Physical examination was within normal limits, except for left torsional low-amplitude nystagmus, which started after a few seconds of head-hanging position and lasted for approximately $1.5 \mathrm{~min}$ in the left ear. Left posterior semicircular canal BPPV was diagnosed, and Epley maneuver was performed. Three days later, patient was re-evaluated, and left Dix-Hallpike test was found to be positive again. Epley maneuver was repeated. A week later, she was disease-free. Patient had no further BPPV attacks until the end of pregnancy.

\section{Case-4}

A 33-year-old pregnant woman visited our clinic with a complaint of vertigo, especially when getting out of bed. She had no additional audiovestibular symptoms such as ear pain, hearing loss, or ear fullness. She was 12 weeks pregnant and had no co-morbid pathologies. No pregnancy-related risk factors were present. Her physical examination was within normal limits, except for Dix-Hallpike test, in which vertigo and right horizontal rotatory nystagmus was observed after $5 \mathrm{~s}$ of latency and lasted for $15 \mathrm{~s}$. Epley maneuver was performed. A week later, the patient was disease-free. She was followed untill the termination of labor, and no further attacks were observed.

Informed consents were obtained from all the patients involved in this study.

Otorhinolaryngologic evaluation of patients was conducted by different clinicians at our clinic. Additionally, the vestibular tests were performed by another clinician, who was blinded to the study.

\section{Discussion}

Benign paroxysmal positional vertigo has various etiologies, but hormonal abnormalities or hormonal changes as predisposing factors are not clarified sufficiently in the literature. Hormonal alterations during menstrual cycle, gestation, and menopause induce various homeostatic, metabolic effects. Various theories regarding estrogen effects have been proposed. Estrogen receptors were found in the inner ears of normal mice (7). They have been detected, especially in the spiral ganglion and stria vascularis, which are important in hearing transmission and inner ear homeostasis (8). Estrogen alterations are considered to either impair endolymphatic fluid electrolyte concentration, leading to degeneration of otoconial fibers, or induce endolymphatic $\mathrm{pH}$ liabilities, causing otoconial degeneration (1). Estrogen is believed to affect endolymph ionic and anionic homeostasis by regulating ion and anion channels (9, 10). Furthermore, it is assumed that estrogen induces vascular supply to the macula and otoconia due to varied glucose and lipid metabolism (1).

Kilicdag et al. (11) studied the effects of estrogen therapy on hearing in postmenopausal women. They found that both estrogen and hormone therapy (combined estrogen and progesterone) groups had better hearing levels than the control groups. They also found that the estrogen therapy group had significantly better hearing thresholds at low frequencies than the hormone therapy group. They postulated that, unlike estrogen, progesterone may have negative effects on low-frequency hearing levels. 
Ogun et al. (12) investigated the effects of menopause in a large cohort of patients diagnosed with BPPV. Their study showed that $48.1 \%$ of female patients with BPPV experienced their first BPPV attack after menopause. The increased BPPV prevalence with age has previously been well documented and this is higher in females than in males. They also suggested that low and fluctuating estrogen levels may lead to otoconial degeneration.

Estrogen and progesterone levels show variations during the time course of pregnancy. The hormonal impacts on the physiology of the inner ear in each trimester of gestation cycle remain unclear.

In our study, three cases were diagnosed during the late trimesters, when estrogen levels are relatively low and progesterone levels are high. This result corresponds to the data in the literature regarding the role of estrogen in inner ear pathologies. Additionally, hormonal instabilities or alterations, as a cause for BPPV is reconsidered once again with this group of patients. Prolonged bed rest may be another cause for BPPV in pregnant women. Three of our patients were diagnosed during the late gestational weeks (second and third cases had initial symptoms during the second trimester, but both mentioned that the severity increased in proportion with gestational week), when sleeping and daily activities were restricted. Furthermore, pregnant women are usually advised to sleep on their left sides, which may be another risk factor for BPPV. However, all four cases were maintaining a normal gestational period. They had no risk factors that would require additional prolonged bed stay.

Recently, there were reports that calcium and vitamin D metabolism disorders are risk factors for BPPV. Calcium and vitamin D metabolism is usually affected in pregnancy, especially in the late trimesters due to the rapid growth of the fetus. This may be another risk factor for pregnant women suffering from BPPV (13). However, according to the guidelines of American College of Obstetricians and Gynecologists, vitamin D supplementation should only be recommended to pregnant women at an increased risk (14). Hence, routine prenatal screening of vitamin D and serum calcium levels were not conducted by the obstetricians at our medical center. Additionally, there is no consensus on the optimal level and the upper limit of the supplemental doses of these parameters in pregnancy (14). Due to these recent findings of the role of vitamin D metabolism in BPPV, serum vitamin $\mathrm{D}$ and calcium levels were investigated in the fourth case, and vitamin D level was found to be low. The patient was referred to her gynecologist for supplementation therapy. This was the only case with recurrent BPPV. Low vitamin D levels may have induced BPPV attacks in this patient; however, further studies in larger series are required to evaluate this association.

\section{Conclusion}

Although the association between BPPV and gestation remains uncertain, to the best of our knowledge, there is very limited data in the English literature on this subject.

Neurotologists and gynecologists should be aware of pregnant women with vertigo. To improve the quality of life quality during gestation, clinicians should consider this pathology in their differential diagnosis, which will help avoid vertigo-related undesirable conditions that may compromise both maternal and fetal status.

Is pregnancy an independent risk factor of $\mathrm{BPPV}$, or do the numbers of metabolic, functional, or emotional changes during pregnancy serve as separate risk factors in this pathology? The authors hope this case series will increase the attempts for further clinical trials providing data for this question.

Informed Consent: Written informed consent was obtained from patients who participated in this study.

Peer-review: Externally peer-reviewed.

Author Contributions: Concept - K.Ç.; Design - K.Ç.; Supervision E.A.; Resource - K.Ç., N.Ç.; Materials - K.Ç., N.Ç.; Data Collection and/or Processing - K.Ç.; Analysis and/or Interpretation - K.Ç., E.A.; Literature Search K.Ç.; Writing - K.Ç.; Critical Reviews - E.A.

Conflict of Interest: No conflict of interest was declared by the authors.

Financial Disclosure: The authors declared that this study has received no financial support.

\section{References}

1. Giacomini PG, Napolitano B, Alessandrini M, Di Girolamo S, Magrini A. Recurrent paroxysmal positional vertigo related to oral contraceptive treatment. Gynecol Endocrinol 2006; 22: 5-8. [CrossRef]

2. Aydin E, Akman K, Yerli H, Ozluoglu LN. Benign paroxysmal positional vertigo after radiologic scanning: a case series. J Med Case Rep 2008; 2: 92. [CrossRef]

3. Kansu L, Avci S, Yilmaz I, Ozluoglu LN. Long-term follow-up of patients with posterior canal benign paroxysmal positional vertigo. Acta Otolaryngol 2010; 130: 1009-12. [CrossRef]

4. Epley JM. The canalith repositioning procedure: for treatment of benign paroxysmal positional vertigo. Otolaryngol Head Neck Surg 1992; 107: 399-404. [CrossRef]

5. Kansu L, Aydin E, Gulsahi K. Benign paroxysmal positional vertigo after nonotologic surgery: case series. J Maxillofac Oral Surg 2015; 14: 113-5. [CrossRef]

6. Koc EA, Koc B, Eryaman E, Ozluoglu LN. Benign paroxysmal positional vertigo following septorhinoplasty. J Craniofac Surg 2013; 24: 89-90. [CrossRef]

7. Stenberg AE, Wang H, Sahlin L, Stierna P, Enmark E, Hultcrantz M. Estrogen receptors alpha and beta in the inner ear of the 'Turner mouse' and an estrogen receptor beta knockout mouse. Hear Res 2002; 166: 1-8. [CrossRef]

8. Pettersson K, Gustafsson JA. Role of estrogen receptor beta in estrogen action. Annu Rev Physiol 2001; 63: 165-92. [CrossRef] 
9. Chen J, Nathans J. Estrogen-related receptor beta/NR3B2 controls epithelial cell fate and endolymph production by the stria vascularis. Dev Cell 2007; 13: 325-37. [CrossRef]

10. Lee JH, Marcus DC. Estrogen acutely inhibits ion transport by isolated stria vascularis. Hear Res 2001; 158: 123-30. [CrossRef]

11. Kilicdag EB, Yavuz H, Bagis T, Tarim E, Erkan AN, Kazanci F. Effects of estrogen therapy on hearing in postmenopausal women. Am J Obstet Gynecol 2004; 190: 77-82. [CrossRef]
12. Ogun OA, Büki B, Cohn ES, Janky KL, Lundberg YW. Menopause and benign paroxysmal positional vertigo. Menopause 2014; 21: 886-9. [CrossRef]

13. Talaat HS, Abuhadied G, Talaat AS, Abdelaal MS. Low bone mineral density and vitamin $\mathrm{D}$ deficiency in patients with benign positional paroxysmal vertigo. Eur Arch Otorhinolaryngol 2015; 272: 2249-53. [CrossRef]

14. ACOG Committee on Obstetric Practice. ACOG Committee Opinion No. 495: Vitamin D: Screening and supplementation during pregnancy. Obstet Gynecol 2011; 118: 197-8. [CrossRef] 